\title{
A damped algorithm for the split feasibility and fixed point problems
}

\author{
Cun-lin $\mathrm{Li}^{1}$, Yeong-Cheng $\mathrm{Liou}^{2}$ and Yonghong $\mathrm{YaO}^{3 *}$
}

"Correspondence:

yaoyonghong@aliyun.com

${ }^{3}$ Department of Mathematics,

Tianjin Polytechnic University,

Tianjin, 300387, China

Full list of author information is

available at the end of the article

\begin{abstract}
The purpose of this paper is to study the split feasibility problem and the fixed point problem. We suggest a damped algorithm. Convergence theorem is proven. MSC: $47 \mathrm{~J} 25 ; 47 \mathrm{H} 09 ; 65 \mathrm{~J} 15 ; 90 \mathrm{C} 25$

Keywords: split feasibility problem; fixed point problem; nonexpansive mapping; damped algorithm
\end{abstract}

\section{Introduction}

Let $C$ and $Q$ be two closed convex subsets of two Hilbert spaces $H_{1}$ and $H_{2}$, respectively, and let $A: H_{1} \rightarrow H_{2}$ be a bounded linear operator. Finding a point $x^{*}$ satisfies

$$
x^{*} \in C \text { and } A x^{*} \in Q \text {. }
$$

This problem, referred to as the split problem, has been studied by some authors. See, e.g., [1-8] and [9]. Some algorithms for solving (1.1) have been presented. One is Byrne's CQ algorithm [1]

$$
x_{n+1}=P_{C}\left(x_{n}-\tau A^{*}\left(I-P_{Q}\right) A x_{n}\right), \quad n \in \mathbb{N},
$$

where $\tau \in\left(0, \frac{2}{L}\right)$ with $L$ being the largest eigenvalue of the matrix $A^{*} A, I$ is the unit matrix or operator, and $P_{C}$ and $P_{Q}$ denote the orthogonal projections onto $C$ and $Q$, respectively. Motivated by Byrne's CQ algorithm, Xu [6] suggested a single step regularized method

$$
x_{n+1}=P_{C}\left(\left(1-\alpha_{n} \gamma_{n}\right) x_{n}-\gamma_{n} A^{*}\left(I-P_{Q}\right) A x_{n}\right), \quad n \in \mathbb{N} .
$$

Very recently, Dang and Gao [5] introduced the following damped projection algorithm

$$
x_{n+1}=\left(1-\beta_{n}\right) x_{n}+\beta_{n} P_{C}\left(\left(1-\alpha_{n}\right)\left(x_{n}-\tau A^{*}\left(I-P_{Q}\right) A x_{n}\right)\right), \quad n \in \mathbb{N} .
$$

If every closed convex subset of a Hilbert space is the fixed point set of its associating projection, then the split feasibility problem becomes a special case of the split common fixed point problem of finding a point $x^{*}$ with the property

$$
x^{*} \in \operatorname{Fix}(U) \text { and } A x^{*} \in \operatorname{Fix}(T) .
$$

๑ 2013 Li et al.; licensee Springer. This is an Open Access article distributed under the terms of the Creative Commons Attribution License (http://creativecommons.org/licenses/by/2.0), which permits unrestricted use, distribution, and reproduction in any medium, provided the original work is properly cited. 
This problem was first introduced by Censor and Segal [10], who invented an algorithm, which generates a sequence $\left\{x_{n}\right\}$ according to the iterative procedure

$$
x_{n+1}=U\left(x_{n}-\gamma A^{*}(I-T) A x_{n}\right), \quad n \in \mathbb{N} .
$$

Recently, Cui, Su and Wang [11] extended the damped projection algorithm to the split common fixed point problems. For some related work, please refer to [12] and [13, 14].

Motivated by these results, the purpose of this paper is to study the following split feasibility problem and fixed point problem

$$
\text { Find } x^{*} \in C \cap \operatorname{Fix}(T) \text { such that } A x^{*} \in Q \cap \operatorname{Fix}(S) \text {, }
$$

where $S: Q \rightarrow Q$ and $T: C \rightarrow C$ are two nonexpansive mappings. We suggest a damped algorithm for solving (1.3). Convergence theorem is proven.

\section{Preliminaries}

Let $H$ be a real Hilbert space with the inner product $\langle\cdot, \cdot\rangle$ and the norm $\|\cdot\|$, respectively. Let $C$ be a nonempty closed convex subset of $H$.

Definition 2.1 A mapping $T: C \rightarrow C$ is called nonexpansive if

$$
\|T x-T y\| \leq\|x-y\|
$$

for all $x, y \in C$.

We will use $\operatorname{Fix}(T)$ to denote the set of fixed points of $T$, that is, $\operatorname{Fix}(T)=\{x \in C: x=T x\}$.

Definition 2.2 We call $P_{C}: H \rightarrow C$ the metric projection if for each $x \in H$

$$
\left\|x-P_{C}(x)\right\|=\inf \{\|x-y\|: y \in C\} .
$$

It is well known that the metric projection $P_{C}: H \rightarrow C$ is characterized by

$$
\left\langle x-P_{C}(x), y-P_{C}(x)\right\rangle \leq 0
$$

for all $x \in H, y \in C$. From this, we can deduce that $P_{C}$ is firmly-nonexpansive, that is,

$$
\left\|P_{C}(x)-P_{C}(y)\right\|^{2} \leq\left\langle x-y, P_{C}(x)-P_{C}(y)\right\rangle
$$

for all $x, y \in H$. Hence $P_{C}$ is also nonexpansive.

It is well known that in a real Hilbert space $H$, the following two equalities hold

$$
\|t x+(1-t) y\|^{2}=t\|x\|^{2}+(1-t)\|y\|^{2}-t(1-t)\|x-y\|^{2}
$$

for all $x, y \in H$ and $t \in[0,1]$, and

$$
\|x+y\|^{2}=\|x\|^{2}+2\langle x, y\rangle+\|y\|^{2}
$$


for all $x, y \in H$. It follows that

$$
\|x+y\|^{2} \leq\|x\|^{2}+2\langle y, x+y\rangle
$$

for all $x, y \in H$.

Lemma 2.3 [15] Let $C$ be a closed convex subset of a real Hilbert space $H$, and let $S: C \rightarrow C$ be a nonexpansive mapping. Then, the mapping $I-S$ is demiclosed. That is, if $\left\{x_{n}\right\}$ is a sequence in $C$ such that $x_{n} \rightarrow x^{*}$ weakly and $(I-S) x_{n} \rightarrow y$ strongly, then $(I-S) x^{*}=y$.

Lemma 2.4 [16] Assume that $\left\{a_{n}\right\}$ is a sequence of nonnegative real numbers such that

$$
a_{n+1} \leq\left(1-\gamma_{n}\right) a_{n}+\delta_{n}, \quad n \in \mathbb{N}
$$

where $\left\{\gamma_{n}\right\}$ is a sequence in $(0,1)$, and $\left\{\delta_{n}\right\}$ is a sequence such that

(1) $\sum_{n=1}^{\infty} \gamma_{n}=\infty$;

(2) $\lim \sup _{n \rightarrow \infty} \frac{\delta_{n}}{\gamma_{n}} \leq 0$ or $\sum_{n=1}^{\infty}\left|\delta_{n}\right|<\infty$.

Then $\lim _{n \rightarrow \infty} a_{n}=0$.

\section{Main results}

Let $C$ and $Q$ be two nonempty closed convex subsets of real Hilbert spaces $H_{1}$ and $H_{2}$, respectively. Let $A: H_{1} \rightarrow H_{2}$ be a bounded linear operator with its adjoint $A^{*}$. Let $S$ : $Q \rightarrow Q$ and $T: C \rightarrow C$ be two nonexpansive mappings. We use $\Gamma$ to denote the set of solutions of (1.3), that is, $\Gamma=\left\{x^{*} \mid x^{*} \in C \cap \operatorname{Fix}(T), A x^{*} \in Q \cap \operatorname{Fix}(S)\right\}$. Now, we present our algorithm.

Algorithm 3.1 For $x_{0} \in H_{1}$ arbitrarily, let $\left\{x_{n}\right\}$ be a sequence defined by

$$
x_{n+1}=T P_{C}\left(\left(1-\alpha_{n}\right)\left(x_{n}-\delta A^{*}\left(I-S P_{Q}\right) A x_{n}\right)\right) \text { for all } n \in \mathbb{N} \text {, }
$$

where $\left\{\alpha_{n}\right\}_{n \in \mathbb{N}}$ and $\left\{\beta_{n}\right\}_{n \in \mathbb{N}}$ are two real number sequences in $(0,1)$ and $\delta \in\left(0, \frac{1}{\|A\|^{2}}\right)$.

Theorem 3.2 Suppose $\Gamma \neq \emptyset$. Assume the sequence $\left\{\alpha_{n}\right\}_{n \in \mathbb{N}}$ satisfies three conditions

(C1) $\lim _{n \rightarrow \infty} \alpha_{n}=0$;

(C2) $\sum_{n=1}^{\infty} \alpha_{n}=\infty$;

(C3) $\lim _{n \rightarrow \infty} \frac{\alpha_{n+1}}{\alpha_{n}}=1$.

Then the sequence $\left\{x_{n}\right\}$, generated by algorithm (3.1), converges strongly to $x^{*}=P_{\Gamma}(0)$.

Proof For the convenience, we write $z_{n}=P_{Q} A x_{n}, y_{n}=\left(1-\alpha_{n}\right)\left(x_{n}-\delta A^{*}\left(I-S P_{Q}\right) A x_{n}\right)$ and $u_{n}=P_{C}\left(\left(1-\alpha_{n}\right)\left(x_{n}-\delta A^{*}\left(I-S P_{Q}\right) A x_{n}\right)\right)$ for all $n \in \mathbb{N}$. Thus $u_{n}=P_{C} y_{n}$ for all $n \in \mathbb{N}$.

Let $x^{*}=P_{\Gamma}(0)$. Hence, $x^{*} \in C \cap \operatorname{Fix}(T)$ and $A x^{*} \in Q \cap \operatorname{Fix}(S)$. By the firmlynonexpansivity of $P_{C}$ and $P_{Q}$, we can deduce the following conclusions

$$
\begin{aligned}
& \left\|z_{n}-A x^{*}\right\|=\left\|P_{Q} A x_{n}-P_{Q} A x^{*}\right\| \leq\left\|A x_{n}-A x^{*}\right\|, \\
& \left\|u_{n}-x^{*}\right\|=\left\|P_{C} y_{n}-P_{C} x^{*}\right\| \leq\left\|y_{n}-x^{*}\right\| \\
& \left\|S z_{n}-A x^{*}\right\|^{2} \leq\left\|z_{n}-A x^{*}\right\|^{2} \leq\left\|A x_{n}-A x^{*}\right\|^{2}-\left\|z_{n}-A x_{n}\right\|^{2}, \\
& \left\|u_{n+1}-u_{n}\right\|=\left\|P_{C} y_{n+1}-P_{C} y_{n}\right\| \leq\left\|y_{n+1}-y_{n}\right\|
\end{aligned}
$$


and

$$
\left\|z_{n+1}-z_{n}\right\|=\left\|P_{Q} A x_{n+1}-P_{Q} A x_{n}\right\| \leq\left\|A x_{n+1}-A x_{n}\right\| .
$$

From (3.1) and (3.3), we have

$$
\left\|x_{n+1}-x^{*}\right\|=\left\|T u_{n}-x^{*}\right\| \leq\left\|u_{n}-x^{*}\right\| \leq\left\|y_{n}-x^{*}\right\| .
$$

Using (2.3), we get

$$
\begin{aligned}
\left\|y_{n}-x^{*}\right\|^{2}= & \left\|\left(1-\alpha_{n}\right)\left(x_{n}-x^{*}+\delta A^{*}\left(S z_{n}-A x_{n}\right)\right)-\alpha_{n} x^{*}\right\|^{2} \\
\leq & \left(1-\alpha_{n}\right) \|\left(x_{n}-x^{*}+\delta A^{*}\left(S z_{n}-A x_{n}\right)\left\|^{2}+\alpha_{n}\right\| x^{*} \|^{2}\right. \\
= & \left(1-\alpha_{n}\right)\left[\left\|x_{n}-x^{*}\right\|+\delta^{2}\left\|A^{*}\left(S z_{n}-A x_{n}\right)\right\|^{2}\right. \\
& \left.+2 \delta\left(x_{n}-x^{*}, A^{*}\left(S z_{n}-A x_{n}\right)\right)\right]+\alpha_{n}\left\|x^{*}\right\|^{2} .
\end{aligned}
$$

Since $A$ is a linear operator with its adjoint $A^{*}$, we have

$$
\begin{aligned}
\left\langle x_{n}\right. & \left.-x^{*}, A^{*}\left(S z_{n}-A x_{n}\right)\right\rangle \\
& =\left\langle A\left(x_{n}-x^{*}\right), S z_{n}-A x_{n}\right\rangle \\
& =\left\langle A x_{n}-A x^{*}+S z_{n}-A x_{n}-\left(S z_{n}-A x_{n}\right), S z_{n}-A x_{n}\right\rangle \\
& =\left\langle S z_{n}-A x^{*}, S z_{n}-A x_{n}\right\rangle-\left\|S z_{n}-A x_{n}\right\|^{2} .
\end{aligned}
$$

Again using (2.3), we obtain

$$
\left\langle S z_{n}-A x^{*}, S z_{n}-A x_{n}\right\rangle=\frac{1}{2}\left(\left\|S z_{n}-A x^{*}\right\|^{2}+\left\|S z_{n}-A x_{n}\right\|^{2}-\left\|A x_{n}-A x^{*}\right\|^{2}\right) .
$$

By (3.4), (3.9) and (3.10), we get

$$
\begin{aligned}
\left\langle x_{n}-x^{*}, A^{*}\left(S z_{n}-A x_{n}\right)\right\rangle= & \frac{1}{2}\left(\left\|S z_{n}-A x^{*}\right\|^{2}+\left\|S z_{n}-A x_{n}\right\|^{2}-\left\|A x_{n}-A x^{*}\right\|^{2}\right) \\
& -\left\|S z_{n}-A x_{n}\right\|^{2} \\
\leq & \frac{1}{2}\left(\left\|A x_{n}-A x^{*}\right\|^{2}-\left\|z_{n}-A x_{n}\right\|^{2}+\left\|S z_{n}-A x_{n}\right\|^{2}\right. \\
& \left.-\left\|A x_{n}-A x^{*}\right\|^{2}\right)-\left\|S z_{n}-A x_{n}\right\|^{2} \\
= & -\frac{1}{2}\left\|z_{n}-A x_{n}\right\|^{2}-\frac{1}{2}\left\|S z_{n}-A x_{n}\right\|^{2} .
\end{aligned}
$$

Substituting (3.11) into (3.8), we deduce

$$
\begin{aligned}
\left\|y_{n}-x^{*}\right\|^{2} \leq & \left(1-\alpha_{n}\right)\left[\left\|x_{n}-x^{*}\right\|^{2}+\delta^{2}\|A\|^{2}\left\|S z_{n}-A x_{n}\right\|^{2}\right. \\
& \left.+2 \delta\left(-\frac{1}{2}\left\|z_{n}-A x_{n}\right\|^{2}-\frac{1}{2}\left\|S z_{n}-A x_{n}\right\|^{2}\right)\right]+\alpha_{n}\left\|x^{*}\right\|^{2} \\
= & \left(1-\alpha_{n}\right)\left[\left\|x_{n}-x^{*}\right\|^{2}+\left(\delta^{2}\|A\|^{2}-\delta\right)\left\|S z_{n}-A x_{n}\right\|^{2}\right.
\end{aligned}
$$




$$
\begin{gathered}
\left.-\delta\left\|z_{n}-A x_{n}\right\|^{2}\right]+\alpha_{n}\left\|x^{*}\right\|^{2} \\
\leq\left(1-\alpha_{n}\right)\left\|x_{n}-x^{*}\right\|^{2}+\alpha_{n}\left\|x^{*}\right\|^{2} .
\end{gathered}
$$

It follows from (3.7) that

$$
\begin{aligned}
\left\|x_{n+1}-x^{*}\right\|^{2} & \leq\left\|y_{n}-x^{*}\right\|^{2} \\
& \leq\left(1-\alpha_{n}\right)\left\|x_{n}-x^{*}\right\|^{2}+\alpha_{n}\left\|x^{*}\right\|^{2} \\
& \leq \max \left\{\left\|x_{n}-x^{*}\right\|^{2},\left\|x^{*}\right\|^{2}\right\} .
\end{aligned}
$$

The boundedness of the sequence $\left\{x_{n}\right\}$ yields.

Next, we estimate $\left\|x_{n+1}-x_{n}\right\|$. Set $v_{n}=x_{n}-\delta A^{*}\left(I-S P_{Q}\right) A x_{n}$. According to (2.3) and (3.5), we have

$$
\begin{aligned}
\left\|v_{n+1}-v_{n}\right\|^{2}= & \left\|x_{n+1}-x_{n}+\delta\left[A^{*}\left(S P_{Q}-I\right) A x_{n+1}-A^{*}\left(S P_{Q}-I\right) A x_{n}\right]\right\|^{2} \\
= & \left\|x_{n+1}-x_{n}\right\|^{2}+\delta^{2}\left\|A^{*}\left[\left(S P_{Q}-I\right) A x_{n+1}-\left(S P_{Q}-I\right) A x_{n}\right]\right\|^{2} \\
& +2 \delta\left\langle x_{n+1}-x_{n}, A^{*}\left[\left(S P_{Q}-I\right) A x_{n+1}-\left(S P_{Q}-I\right) A x_{n}\right]\right\rangle \\
\leq & \left\|x_{n+1}-x_{n}\right\|^{2}+\delta^{2}\|A\|^{2}\left\|S z_{n+1}-S z_{n}-\left(A x_{n+1}-A x_{n}\right)\right\|^{2} \\
& +2 \delta\left\langle A x_{n+1}-A x_{n}, S z_{n+1}-S z_{n}-\left(A x_{n+1}-A x_{n}\right)\right\rangle \\
= & \left\|x_{n+1}-x_{n}\right\|^{2}+\delta^{2}\|A\|^{2}\left\|S z_{n+1}-S z_{n}-\left(A x_{n+1}-A x_{n}\right)\right\|^{2} \\
& +2 \delta\left\langle S z_{n+1}-S z_{n}, S z_{n+1}-S z_{n}-\left(A x_{n+1}-A x_{n}\right)\right\rangle \\
& -2 \delta\left\|S z_{n+1}-S z_{n}-\left(A x_{n+1}-A x_{n}\right)\right\|^{2} \\
= & \left\|x_{n+1}-x_{n}\right\|^{2}+\delta^{2}\|A\|^{2}\left\|S z_{n+1}-S z_{n}-\left(A x_{n+1}-A x_{n}\right)\right\|^{2} \\
& +\delta\left(\left\|S z_{n+1}-S z_{n}\right\|^{2}+\left\|S z_{n+1}-S z_{n}-\left(A x_{n+1}-A x_{n}\right)\right\|^{2}\right. \\
& \left.-\left\|A x_{n+1}-A x_{n}\right\|^{2}\right)-2 \delta\left\|S z_{n+1}-S z_{n}-\left(A x_{n+1}-A x_{n}\right)\right\|^{2} \\
= & \left\|x_{n+1}-x_{n}\right\|^{2}+\left(\delta^{2}\|A\|^{2}-\delta\right)\left\|S z_{n+1}-S z_{n}-\left(A x_{n+1}-A x_{n}\right)\right\|^{2} \\
& +\delta\left(\left\|S z_{n+1}-S z_{n}\right\|^{2}-\left\|A x_{n+1}-A x_{n}\right\|^{2}\right) \\
\leq & \left\|x_{n+1}-x_{n}\right\|^{2}+\left(\delta^{2}\|A\|^{2}-\delta\right)\left\|S z_{n+1}-S z_{n}-\left(A x_{n+1}-A x_{n}\right)\right\|^{2} \\
& +\delta\left(\left\|z_{n+1}-z_{n}\right\|^{2}-\left\|A x_{n+1}-A x_{n}\right\|^{2}\right) .
\end{aligned}
$$

Since $\delta \in\left(0, \frac{1}{\|A\|^{2}}\right)$, we derive by virtue of (3.6) and (3.13) that

$$
\left\|v_{n+1}-v_{n}\right\| \leq\left\|x_{n+1}-x_{n}\right\| .
$$

From (3.5) and (3.14), we have

$$
\begin{aligned}
\left\|x_{n+1}-x_{n}\right\| & \leq\left\|y_{n+1}-y_{n}\right\| \\
& =\left\|\left(1-\alpha_{n+1}\right) v_{n+1}-\left(1-\alpha_{n}\right) v_{n}\right\| \\
& =\left\|\left(1-\alpha_{n+1}\right)\left(v_{n+1}-v_{n}\right)+\left(\alpha_{n}-\alpha_{n+1}\right) v_{n}\right\|
\end{aligned}
$$




$$
\begin{aligned}
& \leq\left(1-\alpha_{n+1}\right)\left\|v_{n+1}-v_{n}\right\|+\left|\alpha_{n+1}-\alpha_{n}\right|\left\|v_{n}\right\| \\
& \leq\left(1-\alpha_{n+1}\right)\left\|x_{n+1}-x_{n}\right\|+\left|\alpha_{n+1}-\alpha_{n}\right|\left\|v_{n}\right\| .
\end{aligned}
$$

It follows that

$$
\left\|x_{n+1}-x_{n}\right\| \leq \frac{\left|\alpha_{n+1}-\alpha_{n}\right|}{\alpha_{n+1}}\left\|v_{n}\right\|
$$

This, together with condition (C3), implies that

$$
\lim _{n \rightarrow \infty}\left\|x_{n+1}-x_{n}\right\|=0
$$

That is,

$$
\lim _{n \rightarrow \infty}\left\|x_{n}-T u_{n}\right\|=0
$$

Using the firmly-nonexpansiveness of $P_{C}$, we have

$$
\begin{aligned}
\left\|u_{n}-x^{*}\right\|^{2} & =\left\|P_{C} y_{n}-x^{*}\right\|^{2} \\
& \leq\left\|y_{n}-x^{*}\right\|^{2}-\left\|P_{C} y_{n}-y_{n}\right\|^{2} \\
& =\left\|y_{n}-x^{*}\right\|^{2}-\left\|u_{n}-y_{n}\right\|^{2} .
\end{aligned}
$$

Thus,

$$
\begin{aligned}
\left\|x_{n+1}-x^{*}\right\|^{2} & \leq\left\|u_{n}-x^{*}\right\|^{2} \\
& \leq\left\|y_{n}-x^{*}\right\|^{2}-\left\|u_{n}-y_{n}\right\|^{2} \\
& \leq\left(1-\alpha_{n}\right)\left\|x_{n}-x^{*}\right\|^{2}+\alpha_{n}\left\|x^{*}\right\|^{2}-\left\|u_{n}-y_{n}\right\|^{2} .
\end{aligned}
$$

It follows that

$$
\begin{aligned}
\left\|u_{n}-y_{n}\right\|^{2} & \leq\left\|x_{n}-x^{*}\right\|^{2}-\left\|x_{n+1}-x^{*}\right\|^{2}+\alpha_{n}\left\|x^{*}\right\|^{2} \\
& \leq\left(\left\|x_{n}-x^{*}\right\|+\left\|x_{n+1}-x^{*}\right\|\right)\left\|x_{n+1}-x_{n}\right\|+\alpha_{n}\left\|x^{*}\right\|^{2} .
\end{aligned}
$$

This, together with (3.15) and (C1), implies that

$$
\lim _{n \rightarrow \infty}\left\|u_{n}-y_{n}\right\|=0
$$

Returning to (3.18) and using (3.12), we have

$$
\begin{aligned}
\left\|x_{n+1}-x^{*}\right\|^{2} \leq & \left\|y_{n}-x^{*}\right\|^{2} \\
\leq & \left(1-\alpha_{n}\right)\left\|x_{n}-x^{*}\right\|^{2}+\left(1-\alpha_{n}\right)\left(\delta^{2}\|A\|^{2}-\delta\right)\left\|S z_{n}-A x_{n}\right\|^{2} \\
& -\left(1-\alpha_{n}\right) \delta\left\|z_{n}-A x_{n}\right\|^{2}+\alpha_{n}\left\|x^{*}\right\|^{2} .
\end{aligned}
$$


Hence,

$$
\begin{aligned}
& \left(1-\alpha_{n}\right)\left(\delta-\delta^{2}\|A\|^{2}\right)\left\|S z_{n}-A x_{n}\right\|^{2}+\left(1-\alpha_{n}\right) \delta\left\|z_{n}-A x_{n}\right\|^{2} \\
& \quad \leq\left\|x_{n}-x^{*}\right\|^{2}-\left\|x_{n+1}-x^{*}\right\|^{2}+\alpha_{n}\left\|x^{*}\right\|^{2} \\
& \quad \leq\left(\left\|x_{n}-x^{*}\right\|+\left\|x_{n+1}-x^{*}\right\|\right)\left\|x_{n+1}-x_{n}\right\|+\alpha_{n}\left\|x^{*}\right\|^{2}
\end{aligned}
$$

which implies that

$$
\lim _{n \rightarrow \infty}\left\|S z_{n}-A x_{n}\right\|=\lim _{n \rightarrow \infty}\left\|z_{n}-A x_{n}\right\|=0 .
$$

So,

$$
\lim _{n \rightarrow \infty}\left\|S z_{n}-z_{n}\right\|=0
$$

Note that

$$
\begin{aligned}
\left\|y_{n}-x_{n}\right\| & =\left\|\delta A^{*}\left(S P_{Q}-I\right) A x_{n}+\alpha_{n} v_{n}\right\| \\
& \leq \delta\|A\|\left\|S z_{n}-A x_{n}\right\|+\alpha_{n}\left\|v_{n}\right\| .
\end{aligned}
$$

It follows from (3.20) that

$$
\lim _{n \rightarrow \infty}\left\|x_{n}-y_{n}\right\|=0
$$

From (3.16), (3.19) and (3.22), we get

$$
\lim _{n \rightarrow \infty}\left\|x_{n}-T x_{n}\right\|=0 .
$$

Now, we show that

$$
\limsup _{n \rightarrow \infty}\left\langle x^{*}, y_{n}-x^{*}\right\rangle \geq 0 \text {. }
$$

Choose a subsequence $\left\{y_{n_{i}}\right\}$ of $\left\{y_{n}\right\}$ such that

$$
\limsup _{n \rightarrow \infty}\left\langle x^{*}, y_{n}-x^{*}\right\rangle=\lim _{i \rightarrow \infty}\left\langle x^{*}, y_{n_{i}}-x^{*}\right\rangle
$$

Since the sequence $\left\{y_{n_{i}}\right\}$ is bounded, we can choose a subsequence $\left\{y_{n_{i_{j}}}\right\}$ of $\left\{y_{n_{i}}\right\}$ such that $y_{n_{i_{j}}} \rightarrow z$. For the sake of convenience, we assume (without loss of generality) that $y_{n_{i}} \rightarrow z$. Consequently, we derive from the above conclusions that

$$
x_{n_{i}} \rightarrow z, \quad u_{n_{i}} \rightarrow z, \quad A x_{n_{i}} \rightarrow A z \quad \text { and } \quad z_{n_{i}} \rightarrow A z .
$$

By the demiclosed principle of the nonexpansive mappings $S$ and $T$ (see Lemma 2.3), we deduce that $z \in \operatorname{Fix}(T)$ and $A z \in \operatorname{Fix}(S)$ (according to (3.23) and (3.21), respectively). Note that $u_{n_{i}}=P_{C} y_{n_{i}} \in C$ and $z_{n_{i}}=P_{Q} A x_{n_{i}} \in Q$. From (3.25), we deduce $z \in C$ and $A z \in Q$. 
To this end, we deduce that $z \in C \cap \operatorname{Fix}(T)$ and $A z \in Q \cap \operatorname{Fix}(S)$. That is to say, $z \in \Gamma$. Therefore,

$$
\begin{aligned}
\limsup _{n \rightarrow \infty}\left\langle x^{*}, y_{n}-x^{*}\right\rangle & =\lim _{i \rightarrow \infty}\left\langle x^{*}, y_{n_{i}}-x^{*}\right\rangle \\
& =\lim _{i \rightarrow \infty}\left\langle x^{*}, z-x^{*}\right\rangle \\
& \geq 0 .
\end{aligned}
$$

Finally, we prove that $x_{n} \rightarrow x^{*}$. From (3.1), we have

$$
\begin{aligned}
\left\|x_{n+1}-x^{*}\right\|^{2} & \leq\left\|y_{n}-x^{*}\right\|^{2} \\
& =\left\|\left(1-\alpha_{n}\right)\left(v_{n}-x^{*}\right)-\alpha_{n} x^{*}\right\|^{2} \\
& \leq\left(1-\alpha_{n}\right)\left\|v_{n}-x^{*}\right\|^{2}-2 \alpha_{n}\left(x^{*}, y_{n}-x^{*}\right\rangle \\
& \leq\left(1-\alpha_{n}\right)\left\|x_{n}-x^{*}\right\|^{2}-2 \alpha_{n}\left(x^{*}, y_{n}-x^{*}\right\rangle .
\end{aligned}
$$

Applying Lemma 2.4 and (3.26) to (3.27), we deduce that $x_{n} \rightarrow x^{*}$. The proof is completed.

\section{Competing interests}

The authors declare that they have no competing interests.

\section{Authors' contributions}

All authors read and approved the final manuscript.

\section{Author details}

${ }^{1}$ School of Management, Beifang University of Nationalities, Yinchuan, 750021, China. ${ }^{2}$ Department of Information Management, Cheng Shiu University, Kaohsiung, 833, Taiwan. ${ }^{3}$ Department of Mathematics, Tianjin Polytechnic University, Tianjin, 300387, China.

\section{Acknowledgements}

Cun-lin Li was supported in part by NSFC 71161001-G0105. Yeong-Cheng Liou was supported in part by NSC 101-2628-E-230-001-MY3 and NSC 101-2622-E-230-005-CC3. Yonghong Yao was supported in part by NSFC 11071279, NSFC 71161001-G0105 and LQ13A010007.

\section{Received: 27 May 2013 Accepted: 29 July 2013 Published: 14 August 2013}

\section{References}

1. Byrne, C: Iterative oblique projection onto convex subsets and the split feasibility problem. Inverse Probl. 18, 441-453 (2002)

2. Censor, Y, Elfving, T: A multiprojection algorithm using Bregman projections in a product space. Numer. Algorithms 8 221-239 (1994)

3. Ceng, LC, Ansari, QH, Yao, JC: An extragradient method for split feasibility and fixed point problems. Comput. Math. Appl. 64, 633-642 (2012)

4. Wang, F, Xu, HK: Approximating curve and strong convergence of the $C Q$ algorithm for the split feasibility problem. J. Inequal. Appl. 2010, Article ID 102085 (2010)

5. Dang, Y, Gao, Y: The strong convergence of a KM-CQ-like algorithm for a split feasibility problem. Inverse Probl. 27, $015007(2011)$

6. Xu, HK: Iterative methods for the split feasibility problem in infinite-dimensional Hilbert spaces. Inverse Probl. 26, $105018(2010)$

7. Yao, Y, Wu, J, Liou, YC: Regularized methods for the split feasibility problem. Abstr. Appl. Anal. 2012, Article ID 140679 (2012)

8. Yao, Y, Kim, TH, Chebbi, S, Xu, HK: A modified extragradient method for the split feasibility and fixed point problems. J. Nonlinear Convex Anal. 13, 383-396 (2012)

9. Yao, Y, Postolache, M, Liou, YC: Strong convergence of a self-adaptive method for the split feasibility problem. Fixed Point Theory Appl. 2013, 201 (2013)

10. Censor, Y, Segal, A: The split common fixed point problem for directed operators. J. Convex Anal. 16, 587-600 (2009)

11. Cui, H, Su, M, Wang, F: Damped projection method for split common fixed point problems. J. Inequal. Appl. 2013, 123 (2013). doi:10.1186/1029-242X-2013-123 
12. Moudafi, A: A note on the split common fixed-point problem for quasi-nonexpansive operators. Nonlinear Anal. 74, 4083-4087 (2011)

13. He, ZH: The split equilibrium problems and its convergence algorithms. J. Inequal. Appl. 2012, 162 (2012)

14. He, ZH, Du, WS: On hybrid split problem and its nonlinear algorithms. Fixed Point Theory Appl. 2013, $47(2013)$

15. Geobel, K, Kirk, WA: Topics in Metric Fixed Point Theory. Cambridge Studies in Advanced Mathematics, vol. 28. Cambridge University Press, Cambridge (1990)

16. Xu, HK: Iterative algorithms for nonlinear operators. J. Lond. Math. Soc. 66, 240-256 (2002)

doi:10.1186/1029-242X-2013-379

Cite this article as: Li et al.: A damped algorithm for the split feasibility and fixed point problems. Journal of Inequalities and Applications 2013 2013:379.

\section{Submit your manuscript to a SpringerOpen ${ }^{\circ}$} journal and benefit from:

- Convenient online submission

Rigorous peer review

- Immediate publication on acceptance

- Open access: articles freely available online

- High visibility within the field

- Retaining the copyright to your article 